\title{
JURISPRUDENCIA INGLESA COMENTADA ¿POR QUÉ NO TE CALLAS?: RESPONSABILIDAD CIVIL POR RUIDOS MOLESTOS Y OTRAS INTERFERENCIAS CON EL USO Y GOCE DE UN INMUEBLE. COMENTARIO DE LA SENTENCIA DE LA CORTE SUPREMA DEL REINO UNIDO EN LA CAUSA COVENTRY AND OTHERS V LAWRENCE AND ANOTHER
}

\author{
Arturo Ibáñez León \\ University of Oxford
}

\section{INTRODUCCIÓN}

$\mathrm{Al}$ abogado formado en la tradición del Derecho Continental ${ }^{1}$ que se adentre en las normas sobre responsabilidad extracontractual de un sistema legal perteneciente a la tradición del Common Law $^{2}$, como es el caso del

${ }^{1}$ La tradición del Derecho Continental comprende a aquellos sistemas legales que reconocen sus orígenes fundamentalmente en el Derecho Romano. A dicha tradición también se le denomina, en particular en los países de la tradición del Common Law, tradición del Derecho Civil (civil law). Al respecto, ZwEIGERT y Köтz (1998), pp. 63-73

${ }^{2}$ La expresión Common Law tiene más de una acepción. Así, Common Law puede referirse al conjunto de principios y reglas de creación judicial sobre la base del precedente. También puede significar el cuerpo de reglas establecidas por los tribunales de Common Law, en oposición a las reglas de equidad (equity law), cuyo origen se encuentra en los pronunciamientos de las courts of Chancery. En ocasiones se habla de Common Law para referirse a los principios generales del derecho o principios fundamentales del Derecho inglés. Por último, la expresión Common Law alude
Derecho inglés ${ }^{3}$, probablemente le llamarán de inmediato la atención dos rasgos preliminares. En primer lugar, le saltará a la vista el que las normas constitutivas de lo que conocemos como derecho de la responsabilidad extracontractual (lo que en el derecho inglés se denomina tort law o law of torts), no se encuentren contenidas en disposiciones codificadas sino que principalmente en sentencias de los tribunales superiores de justicia, las cuales, bajo determinadas condiciones, son obligatorias para los tribunales inferiores. El conjunto de dichas decisiones judiciales es lo que se denomina el Case Law o Derecho

también a una cultura legal, a una particular actitud respecto del derecho y de los problemas legales. Véase BELL (2013), pp. 10-11.

${ }^{3} \mathrm{El}$ sistema legal inglés (que rige en Inglaterra y Gales) es uno de los tres sistemas legales que coexisten en la unidad política que se denomina Reino Unido de la Gran Bretaña e Irlanda del Norte. Los otros dos son el sistema legal de Irlanda del Norte y el de Escocia. Al respecto, Le Sueur (2009), p. 12. 
de Casos. Se trata, en consecuencia, de un derecho que es, fundamentalmente, de creación judicial ${ }^{4}$. Ello porque son los tribunales superiores de justicia los que la mayoría de las veces crean y desarrollan las normas de este derecho de casos, proceso en el cual ocupa un lugar fundamental la doctrina del precedente. ${ }^{5}$

$\mathrm{Y}$, en segundo lugar, le llamará también la atención la ausencia de una regla general relativa a la responsabilidad extracontractual como la que se establece en el artículo 2314 del Código Civil chileno o en los artículos 1382 y 1383 del Código Civil francés. Por el

${ }^{4}$ No todos los torts tienen su origen en decisiones judiciales. También existen torts de creación legal, es decir, torts que tienen su origen en leyes dictadas por el parlamento británico (statutes o Acts of Parliament). Sin embargo, esas leyes serán interpretadas $\mathrm{y}$ aplicadas por decisiones judiciales con autoridad de precedente, las que también formarán parte del derecho de casos. En todo caso, lo que no debe perderse de vista es que, si bien es cierto que el Common Lare, entendido en esta parte como el conjunto de principios y reglas de creación judicial sobre la base del precedente, constituye una importante fuente de Derecho, no es menos cierto que el derecho legislado o Statutory Law (integrado por la les leyes dictadas por el parlamento británico, lo que se denomina legislación primaria; y por las normas dictadas por el Ejecutivo en virtud de una delegación del Parlamento, lo que se denomina legislación secundaria) también constituye una importante fuente del Derecho, al extremo que es jerárquicamente superior a las normas de creación judicial sobre la base del precedente (Common Law). De esta forma, una ley del Parlamento británico puede, por ejemplo, crear un nuevo tort (un statutory tort) o modificar normas de Tort Law contenidas en decisiones judiciales o en leyes. Véase al respecto BELL (2013), pp. 3-35; MCBRIDE y BAGSHAW (2012), pp. 21-23.

${ }^{5}$ Sobre el precedente y sus reglas, véase BeLl (2013), pp. 23-34. contrario, en el Derecho inglés existen una serie de ilícitos civiles (torts) ${ }^{6}$, cada uno de los cuales constituye una causa de acción independiente. De esta forma, la persona que en razón de haber sido víctima de un ilícito civil pretenda obtener algún remedio en particular (generalmente, una indemnización de perjuicios) deberá alegar que ha sido víctima de un tort en particular, para lo cual deberá encuadrar los hechos en alguno de los torts reconocidos por el Derecho ${ }^{7}$. En razón de ser fundamentalmente materia de creación judicial, y de la antigüedad de algunas de las decisiones que revisten la autoridad de precedente en estas materias (algunas de ellas pronunciadas hace cientos de años), no existe claridad absoluta acerca del número total de torts que existen en el Derecho inglés. Sin embargo, sí es claro que hay algunos que revisten especial importancia. Por ejemplo, el tort de negligencia ocupa un lugar predominante en la literatura

${ }^{6}$ Un tort constituye una categoría de ilícito civil. Existen ilícitos civiles que no constituyen un tort como por ejemplo, el incumplimiento de un contrato y los ilicitos en el ámbito de Equity Law. Al respecto, Birks (1995), pp. 31-51.

${ }^{7}$ En el Derecho inglés existen fundamentalmente dos concepciones acerca del Derecho de torts. Para algunos el derecho torts se ocupa de definir en qué casos un daño sufrido por una persona debe ser compensado por otra. Con arreglo a este modelo, la esencia del derecho de torts es la existencia de un daño. Según otra concepción, el derecho de torts se ocupa de la obligación secundaria a que da origen la violación de una obligación primaria, la cual es correlativa a un derecho subjetivo privado. De acuerdo con esta concepción, la esencia del derecho de torts es la violación de derechos subjetivos privados. Véase al respecto STEVEns (2009), pp. 1-3. 
jurídica inglesa relativa al derecho de torts. Dicho tort probablemente cubre la mayoría de las hipótesis que en el Derecho chileno son constitutivas de responsabilidad extracontractual con arreglo a la norma del artículo 2314 del Código Civil.

Entre los torts de especial relevancia en el derecho inglés cabe también mencionar el tort de private nuisance. Al respecto, es preciso señalar al menos cuatro puntos básicos para hacerse una idea general acerca de la naturaleza y alcance de este tort. En primer lugar, se trata de uno de los que protege la propiedad ${ }^{8}$, o mejor dicho, los derechos o intereses propietarios ${ }^{9}$. En

${ }^{8}$ Otros torts que también protegen los derechos propietarios en inmuebles son el de trespass to land y el de negligencia. Véase Mc FARLANe (2008), pp. 651-652.

${ }^{9}$ No es este el lugar para ahondar en las complejidades de las normas del Derecho inglés que constituirían lo que en el Derecho chileno llamamos derecho de bienes. Bastará decir que en materia de inmuebles existen varios derechos propietarios (que vendrían a representar lo que en Derecho chileno son los derechos reales) los que se agrupan en torno a dos conceptos fundamentales: estates en inmuebles (que consisten en derechos de control exclusivo de un inmueble) e intereses en inmuebles (que consisten en un derecho a un uso limitado sobre un inmueble). Son estates en inmuebles el freehold (lo más cercano a la noción de dominio en el Derecho chileno) y el lease (una especie de arrendamiento de largo plazo). Son intereses en inmuebles el easement (lo que en el Derecho chileno sería una servidumbre) y el charge (lo que en el derecho chileno sería una garantía real). Cuando en el Derecho inglés se habla de ownership (dominio) se está hablando de situaciones en la que existe un derecho de control exclusivo, lo que tiene lugar en el caso del freehold o de un lease. Asimismo, cuando en el texto de este comentario se hable de derechos propietarios, se deberá entender que dicha expresión comprende en general el freehold, el lease, el ease- el caso de private nuisance, se protegen los derechos o intereses propietarios sobre un inmueble. Por ello, en la literatura jurídica inglesa se califica a este tort como un against land (en contra de la tierra, es decir, un ilícito civil consistente en la violación de derechos o intereses propietarios sobre un inmueble ${ }^{10}$. Este rasgo explica, por ejemplo, las reglas sobre legitimación activa que gobiernan este tort: solo las personas que sean titulares de derechos propietarios sobre el inmueble respectivo tendrán la calidad de víctima de este torty, consecuentemente, estarán habilitadas para demandar en private nuisance ${ }^{11}$. En segundo lugar, el núcleo del tort de private nuisance consiste en una interferencia sustancial con derechos propietarios sobre un inmueble. El carácter sustancial o inaceptable de las interferencias es lo que constituye la ilicitud en este tort. Por ello, lo clave para que se configure una nuisance es que se trate

ment y el charge. A propósito es fundamental McFarlane (2006), en especial p. 341 y ss.

${ }^{10}$ Nolan (2014), pp. 459-490.

${ }^{11}$ Por la misma razón, los daños accionables son el daño físico al inmueble, la interferencia con el confort y conveniencia en el uso y goce del inmueble. En la medida que sean una consecuencia de dichos daños que se puedan prever de manera razonable son también accionables los daños a intereses económicos y a bienes muebles destinados en el inmueble. Desde este punto de vista, se argumenta que los daños físicos a personas serían también accionables en la medida que sean una consecuencia razonablemente previsible del daño físico a la propiedad o de la interferencia con el confort y conveniencia en el uso y goce del inmueble, pero ello en general no es aceptado. Véase al respecto McBride y Bagshaw (2012), pp. 459-464; Nolan (2014), pp. $477-480$ 
de interferencias sustanciales ${ }^{12}$. No toda interferencia es constitutiva de nuisance, puesto que la vida en sociedad implica el tener que aceptar ciertas interferencias, las que por tal motivo (por ser consustanciales a la vida en sociedad) son consideradas aceptables. Dichas interferencias pueden afectar el uso y goce de un inmueble, es decir, interferencias con el valor de amenidad del inmueble (por ejemplo, emanaciones de olores, ruidos, etc), o en un daño físico causado al inmueble, en tanto que dicho daño reduce el valor de amenidad del inmueble $^{13}$. En tercer lugar, el tort de

${ }^{12}$ Gran parte de las decisiones judiciales y de la literatura jurídica habla de interferencias que no sean razonables. Por ejemplo, McBride y Bagshaw (2012), pp. 415-416; Murphy (2010), pp. 5-6. Pero otros, a fin de evitar confusiones con el tort de negligencia y la idea de culpa, prefieren hablar solo de interferencia sustancial, por ejemplo, Nolan (2014), pp. 468-473. De esta forma, los legitimados pasivo son los que crean, autorizan, adoptan o continuán el estado de cosas que produce la interferencia sustancial. Cuando se habla de "estado de cosas" se quiere dejar en claro que, en general, las interferencias sustanciales causadas por un acto único no quedan comprendidas en el tort, aunque ello es discutible. Véase al respecto, McBride y Bagshaw (2012), pp. 440-446. BeEVER (2013) plantea un nuevo entendimiento del derecho de private nuisance, concibiéndolo como un sistema dirigido a priorizar los usos en conflicto, privilegiando aquellos que son más fundamentales para el entendimiento de lo que es la propiedad.

${ }^{13}$ Las interferencias pueden consistir en emanaciones (que será el caso más común), invasiones de objetos físicos no constitutivas de trespass (siendo el caso típico el de las raíces de un árbol que penetran en el inmueble vecino), obstrucciones (típicamente, interferencias con un easement o derecho de servidumbre), y, aunque discutible, casos de afrenta, esto es, inteferencias con el uso y el goce de un inmueble causados por la afrenta private nuisance constituye lo que se denomina un tort de responsabilidad estricta (strict liability tort). Es decir, el demandado incurrirá en responsabilidad civil por private nuisance aun cuando ejecute diligentemente las actividades constitutivas de nuisance (es decir, pese a que no incurra en culpa $)^{14}$. Y, en cuarto lugar, en lo que tiene relación con los remedios disponibles para el demandante, un lugar relevante ocupan las injunctions, esto es, órdenes judiciales en virtud de las cuales se prohibe continuar con las actividades constitutivas de nuisance (prohibitory injunctions) o se ordena ejecutar determinadas medidas dirigidas a prevenir la causacón de daño futuro (mandatory injunctions).

El presente comentario se ocupa de una sentencia particularmente relevante en materia de private nuisance dictada por la Corte Suprema del Reino Unido (en adelante "la Corte" o "la Corte Suprema")15. Se trata de

o repugnancia que tiene lugar al saber que en un inmueble vecino se ejecutan actividades para un determinado propósito (por ejemplo, un cine pornográfico, un prostíbulo o un sex shop). Véase McBride y Bagshaw (2012), pp. 419-439.

${ }^{14}$ Murphy (2010), pp. 54-57 matiza esta afirmación señalando que private nuisance es un tort de responsabilidad estricta en el sentido que el demandado causante de la interferencia será responsable no obstante haber tomado las precauciones correspondientes para la ejecución de la actividad respectiva (es decir, a pesar de haber sido diligente), pero que ello no excluye afirmar que solo será responsable en la medida que exista otra especie de falta de su parte que hace reprochable su conducta, a saber, que ejecutó una actividad que suponía un riesgo previsible de daño o interferencia.

${ }^{15}$ La Corte Suprema del Reino Unido fue establecida por las normas de la Constitutional Reform Act de 2005, la cual dispuso que a partir 
la sentencia dictada con fecha 26 de febrero de 2014 en la causa Coventry an others $\mathrm{v}$ Lawrence and another. Dos factores en particular explican la relevancia de dicha sentencia. En primer lugar, diversas y cruciales normas en materia de private nuisance (desarrolladas por los propios tribunales a través del Case Law o derecho de casos) fueron debatidas en el pleito $\mathrm{y}$, en segundo lugar, en al menos dos puntos en particular, la Corte Suprema se apartó del precedente y desarrolló las normas existentes en la materia.

El comentario de la referida sentencia consta de tres partes. En una primera parte, se efectuará una síntesis del caso. Se expondrán los hechos relevantes y los pronunciamientos del juez de primera instancia y de la Corte de Apelaciones. En una segunda parte se enunciarán las cuestiones debatidas y se analizará la decisión de la Corte Suprema respecto de cada uno de ellas. Finalmente, en una tercera parte, se formularán alguna conclusiones.

del 1 de octubre de 2009 la nueva Corte sería el tribunal final de apelación y la cúspide de la estructura judicial británica. En su calidad de tribunal final de apelación, se ocupa de materias jurídicas de interés o relavantes para el desarrollo del Derecho. Aunque conoce de apelaciones, sólo se pronuncia respecto del Derecho. Además, la propia Corte es la que determina qué asuntos van a ser conocidos, siempre sobre la base del criterio de la relevancia o interés jurídico de la materia. Hasta el 1 de octubre de 2009 el tribunal final de apelación era el comité de apelaciones de la House of Lords, cuyas funciones judiciales fueron traspasadas a la nueva Corte Suprema.
II. UNA SÍNTESIS DEL CASO

\subsection{Los hechos}

En febrero de 1975 Terry Waters obtuvo una autorización administrativa (planning permission, es decir, una autorización otorgada de conformidad con lo que podría considerarse equivalente en nuestro ordenamiento a las normas de un plan regulador del uso del suelo) para construir en la campiña de Suffolk (en terrenos destinados principalmente a la agricultura) un estadio que albergara una pista de carreras de motocicletas (en adelante "el estadio"). La autorización administrativa permitía que el estadio fuera utilizado para llevar a cabo dichas carreras y para usar sus instalaciones por un plazo de diez años. En 1985 la autorización fue renovada pasando a tener un carácter permanente. El estadio fue construido en 1976 y comenzó a ser usado para carreras de motocicletas, una actividad deportiva eminentemente ruidosa, con ocasionales interrupciones en algunos años (el estadio albergaba a Fen Tigers, un equipo de carreras de motocicletas que participaba en competencias nacionales y que actuaba como local en el estadio. El equipo era de propiedad de la sociedad Fen Tigers Ltd).

En el año 1984 el Terry Waters también comenzó a organizar en el estadio carreras de autómoviles para las cuales no contaba con autorización administrativa (específicamente, stockcar racing y banger racing). $\mathrm{Al}$ cabo de diez años, en 1994, con el fin de regularizar la situación y de conformidad con determinadas normas administra- 
tivas, solicitó un certificado de legalidad del uso o desarrollo existentes. Dicho certificado fue otorgado por la autoridad administrativa en 1997 , sobre la base de que a lo largo de un período de diez años el estadio había albergado cada año veinte eventos de stock car y banger racing (a específicas horas del día), por lo que tal uso había pasado a ser legal de acuerdo con las normas de lo que denominaríamos un plan regulador del uso de suelos urbanos y rurales. Cabe mencionar que en el año 1992 el estadio comenzó también a albergar carreras de galgos.

En mayo de 1992 obtuvo una autorización administrativa (planning permission) para construir una pista o track en la parte trasera del estadio y para albergar en ella carreras de motocross. La autorización fue concedida por un año, renovándose en sucesivas oportunidades. Tanto la autorización original como sus sucesivas renovaciones contemplaban condiciones especificas dirigidas a controlar la frecuencia de los eventos a desarrollarse en la pista o tracky los niveles de ruido producto de dichos eventos. Finalmente, en el año 2002, se otorgó una autorización administrativa de carácter permanente para albergar carreras de motocross, también sujeta a condiciones similares a las establecidas con anterioridad, incluyendo una en virtud de la cual se limitaba el uso de la pista a un cierto número de días y dentro de determinadas horas; y otra en virtud de la cual se establecía un límite máximo de los niveles de ruido.

En octubre de 2005 el estadio fue adquirido por el hijo de Terry Waters, James Waters. Luego de sucesivos ac- tos jurídicos, en el año 2008 el estadio fue adquirido finalmente por David Coventry y su hermano, quienes son los dueños del estadio y lo han operado desde entonces (Fen Tigers Ltd, la sociedad dueña del equipo Fen Tigers, continuó promoviendo carreras de motocicletas a realizarse en el estadio, hasta su disolución, en el año 2010). Por otra parte, Terence Waters es uno de los tres copropietarios de la pista de motocross o track. En el año 2003 la pista o track fue entregada en arrendamiento por el plazo de diez años a la sociedad Moto-Land UK Ltd, la cual ha operado desde entonces las actividades en la pista o track.

A una distancia de alrededor de quinientos sesenta metros del estadio y ochocientos sesenta metros de la pista o track, se ubica una cabaña o casa de campo construida en la década de 1950 (cottage o bungalow) denominada "Fenland". Dicha cabaña tiene alrededor de 0,35 ha de jardín y está rodeada de terrenos destinados a la agricultura en términos tales que el sector residencial más cercano es la pequeña villa de West Row, ubicada a alrededor de milla y media (aproximadamente $2,4 \mathrm{~km}$ ) al sureste de "Fenland" y a alrededor de una milla (cerca de 1,6 km) al sureste del estadio.

Desde el año 1984, los dueños y residentes de "Fenland" eran el señor y la señora Relton. En el año 2006 los Relton vendieron la propiedad a Katherine Lawrence y Raymond Shields (en adelante "los demandantes" o "los apelantes"), quienes se mudaron a la cabaña en el mismo año. Poco después de haber comenzado a residir en "Fenland" los demandantes 
se vieron perturbados por los ruidos provenientes de las carreras de motocross que se llevaban a cabo en la pista o track. En virtud de ello, reclamaron por escrito a Terence Waters y su hijo James, a David Coventry, y a la sociedad Moto Land UK (en adelante "los demandados" o "los apelados") y al gobierno local. Este último, con sujeción a normas administrativas, ordenó la ejecución de trabajos de atenuación de los ruidos, orden que se notificó a los apelados en diciembre de 2007. Los trabajos de atenuación se dilataron más allá del plazo establecido para su realización, siendo ejecutados finalmente en enero de 2009.

En el intertanto, los demandantes habían comenzado a plantear que tanto el estadio como la pista o track estaban siendo usados en una forma tal que constituía una private nuisance, esto es, una interferencia sustancial con su derecho a usar y gozar de "Fenland" producto de los ruidos molestos provenientes de las actividades llevadas a cabo en dichos recintos. Como las conversaciones con los demandados no prosperaron, y considerando, además, que estos retardaron la ejecución de los trabajos de atenuación de ruidos ordenados por la autoridad local, a comienzos de 2008 los demandantes entablaron una acción en private nuisance en contra de los demanddos, pretendiendo como remedio que el tribunal decretara una injunction, esto es, una orden judicial que dispusiera la prohibición de los actos que en concepto de los apelantes eran constitutivos de private nuisance (la emisión de ruidos molestos). No obstante que a comienzos de 2009 los demandados ejecutaron los trabajos de atenuación de ruidos ordenados por la autoridad local, lo demandantes decidieron perseverar en su acción judicial en private nuisance. Por ello, en diciembre de 2009, los demandados presentaron conjuntamente una contestación o defensa pidiendo el rechazo de la demanda.

Por último, cabe mencionar que en abril de 2010 un incendio afectó a "Fenland", sufriendo tal extensión de daños que se volvió inhabitable. Desde esa fecha ni los demandantes ni ninguna otra persona han residido en la cabaña.

\subsection{Los pronunciamientos del juez de primera instancia y de la Corte de Apelaciones}

El juez de primera instancia emitió su pronunciamiento el 4 de marzo de 2011, declarando que los demandados habían cometido private nuisance, por lo que acogió la demanda. Habiendo establecido que los demandados habían incurrido en private nuisance, el juez decretó una injunction u orden judicial en virtud de la cual se dispuso que los demandados no podían ejecutar actividades en el estadio o en la pista que excedieran ciertos niveles de ruido (expresados en decibeles), los cuales determinó considerando la evidencia proporcionada a propósito de los niveles de ruido emitidos en otras pistas de carrera a lo largo del Reino Unido. Además, el juez señaló que los niveles de ruido permitidos debían ser aún más bajos en los atardeceres y durante la noche. En razón de que a la fecha del pronunciamiento de la 
sentencia "Fenland" se encontraba inhabitada, el juez consideró apropiado suspender provisoriamente la injunction decretada. Por último, el juez otorgó a los demandantes una indemnización de perjuicios por los daños ya causados. Con posterioridad a este pronunciamiento, el juez relajó en cierta forma los términos de la injunction decretada. En efecto, en primer lugar, autorizó a los demandados para ejecutar actividades en el estadio y en la pista produciendo niveles de ruido relativamente más altos que aquellos que había fijado con anterioridad, hasta por doce fines de semanas cada año. Asimismo, y con el fin de otorgar un plazo a los demandados para reorganizar sus asuntos y adoptar las medidas pertinentes a fin de cumplir con lo decretado, el juez dispuso que la injunction solo tendría efecto a partir del 1 de enero de 2012, o a partir de la fecha en la que "Fenland" estuviera habilitada nuevamente para uso residencial, si ello tenía lugar en fecha posterior. Por último, el juez concedió a cada parte el derecho a solicitar la modificación de los términos y condiciones de la injunction decretada, derecho que no podía ser ejercido antes del 1 de octubre de 2011.

Los demandados recurrieron en contra de la sentencia y la Corte de Apelaciones acogió el recurso, revocando la sentencia del juez de primera instancia. La Corte de Apelaciones sostuvo que los demandantes no habían podido establecer que las actividades de los demandados en el estadio y en la pista o trackfueran constitutivas de nuisance. En particular, el fallo revocatorio señaló que el juez de primera instancia había incurrido en un error al sostener que para los efectos de evaluar el carácter de la localidad con miras a determinar la existencia de una nuisance no correspondía tomar en cuenta el uso concreto que durante varios años los demandados habían dado al estadio y a la pista o track, contando para ello con una autorización administrativa o con un certificado de legalidad del uso y desarrollo existentes. La Corte concluyó que sí correspondía tomar en cuenta tales factores y que, al tomarlos en cuenta, no se podía sino concluir que los demandantes no habían podido establecer que las actividades de los demandados habían constituído una nuisance.

En contra del fallo de la Corte de Apelaciones recurrieron los demandantes.

\section{El PRONUNCIAMIENTO de la Corte Suprema}

La Corte Suprema, por la unanimidad de sus miembros ${ }^{16}$, acogió la apelación de los demandantes. Si bien cada uno de los miembros del panel que conoció y falló el asunto emitió una opinión escrita, el pronunciamiento principal consta en la opinión escrita de lord David Neuberger ${ }^{17}$, con quien el resto de los miembros concordó en lo sustancial.

La Corte Suprema analizó cinco cuestiones que configuran el núcleo

${ }^{16}$ Un panel de cinco miembros de la Corte conoció y falló la causa: lord David Neuberger (quien presidió el panel), lord Jonathan Mance, lord Anthony Clarke, lord Jonathan Sumption, y lord Robert Carnwath.

${ }^{17}$ Párrafos 1 a 153 del fallo. 
de lo debatido en el pleito y que inciden en puntos claves de las normas relativas al tort de private nuisance. Es preciso indicar dichas cuestiones antes de pasar al análisis de lo resuelto por la Corte.

\subsection{Las cuestiones debatidas}

Como se indicó, el pleito involucró diversas cuestiones relativas a las normas aplicables en materia de private nuisance. Estas cuestiones fueron las siguientes:

a) Si es posible, y en qué medida, un demandado en private nuisance puede alegar que ha adquirido por prescripción el derecho a ejecutar actos que, de no mediar dicha prescripción, serían constitutivos de private nuisance;

b) Si es posible, y en qué medi$\mathrm{da}$, un demandado en private nuisance puede alegar como defensa la circunstancia de que el propio demandante se expuso a sí mismo o "vino a la nuisance" al decidir trasladarse a residir al lugar donde se estaban ejecutando desde hace un tiempo los actos que se alegan como constitutivos de private nuisance;

c) Si es posible, y en qué medida, un demandado en private nuisance puede invocar el uso concreto que él hace de su propio inmueble o instalaciones (uso respecto del cual se alega que es constitutivo de private nuisance) para los efectos de evaluar y determi- nar lo que se denomina "el carácter de la localidad";

d) Si es posible, y en qué medida, el otorgamiento de una autorización administrativa (planning permission) para un determinado uso puede incidir en dos cuestiones:

i) en la determinación de si tal uso es constitutivo de nuisance (es decir, si es posible, y en qué medida, el demandado puede alegar la autorización administrativa como defensa en razón de que dicha autorización permite el uso por el cual precisamente se reclama) y

ii) en la determinación del "carácter de la localidad" (es decir, si es posible que el demandado alegue que la autorización administrativa, en la medida que permitió que su inmueble $\mathrm{u}$ otros inmuebles en la localidad pudieran ser usados para un determinado propósito, cambió efectivamente el "carácter de la localidad") y

e) El enfoque que debe adoptarse por parte del tribunal al decidir si otorga como remedio una injunction o una indemnización de perjuicios; $y$, con respecto a dicha decisión, la relevancia del hecho que exista una autorización administrativa para el uso por el cual se reclama. 


\subsection{La decisión de la Corte Suprema}

a) En cuanto a la posibilidad de adquirir por prescripción el derecho a ejecutar actos que de otro modo serían constitutivos de private nuisance

La Corte Suprema, a diferencia de lo que había resuelto el juez primera instancia, resolvió este punto dejando establecido que es posible adquirir por prescripción el derecho a ejecutar actos que de otro modo serían constitutivos de nuisance, tales como la emisión de ruidos molestos. Al respecto, cuatro puntos son los que cabe destacar.

En primer lugar, lord David Neuberger señala que se encuentra bien establecido que un easement (lo que en el Derecho chileno constituiría una servidumbre real) puede ser adquirido por prescripción. Para tales efectos, es un requisito fundamental que la persona que alega la prescripción demuestre que ha gozado continuamente de lo que constituiría el derecho objeto de la servidumbre por un plazo de al menos veinte años, disfrute que, además, debe ser nec vi, nec clam y nec precario (es decir, sin fuerza, sin clandestinidad, y sin estar basado en la concesión de una autorización por parte del propietario del predio sirviente).

En segundo lugar, si bien es posible adquirir por prescripción una servidumbre real, una cuestión diferente es la de determinar si la ejecución de actos generadores de ruidos molestos constitutivos de nuisance puede en sí misma ser objeto de una servidumbre real ${ }^{18}$. Asimismo, aborda este punto y señala que ello es posible, no siendo un impedimento para arribar a dicha conclusión las particulares características de lo que constituye en este caso la carga o gravamen (los ruidos molestos), en particular, su intermitencia ${ }^{19}$.

En tercer lugar, habiendo establecido que es posible que la emisión de ruidos molestos puede ser objeto de una servidumbre real, pasa a analizar si dicha servidumbre en particular puede ser adquirida mediante prescripción. Es decir, si en este caso se configura o no una excepción a la regla general antes señalada de que es posible adquirir por prescripción un easement (servidumbre real).

En esta parte reconoce la existencia de tres dificultades ${ }^{20}$. La primera de dichas dificultades es que el plazo de veinte años correrá efectivamente en la medida que los ruidos emitidos sean constitutivos de nuisance porque solo a propósito de tales actos el dueño del predio sirviente puede ejercer oposición. De lo contrario, no tendría lugar uno de los fundamentos de la prescripción (el que la ausencia

${ }^{18}$ En el Derecho inglés la lista de los easements (servidumbres reales) no es cerrada. Véase al respecto McFarlane (2008), p. 838 y ss.

${ }^{19} \mathrm{Cf}$. la situación en el Derecho chileno, donde el artículo 882 del Código Civil establece expresamente que las servidumbres discontinuas de todas clases y las servidumbre continuas inaparentes solo pueden adquirirse por medio de un título, agregando que "ni aún el goce inmemorial bastará para constituirlas".

${ }^{20}$ Además de la servidumbre cuyo objetivo es la emisión de ruidos molestos, estas dificultades también existirían respecto de servidumbres que tengan por finalidad el derecho a ejecutar lo que de otro modo sería una nuisance por emisión de vibraciones, humos u olores. 
de oposición por parte del dueño del predio sirviente hace suponer su aquiescencia $)^{21}$. Dicho de otro modo, el plazo se suspenderá cuando los ruidos no superen el nivel que los hace constitutivos de nuisance. Una segunda dificultad será el determinar la extensión de la servidumbre adquirida por prescripción, esto es, el determinar qué niveles de ruido podrán ser emitidos con arreglo a la servidumbre. Esta dificultad surge porque aun cuando durante el periodo de veinte años todos los ruidos emitidos hayan sido constitutivos de nuisance, los mismos habrán presentado variaciones en términos de intensidad y frecuencia. $\mathrm{Y}$ una tercera dificultad consiste en determinar si, después de adquirida la servidumbre, el titular de la misma puede emitir ruidos de un nivel superior a aquellos emitidos durante el periodo de veinte años.

Sostiene, sin embargo, que las tres dificultades mencionadas no constituyen un impedimento insuperable para los efectos de afirmar que es posible adquirir por prescrición el derecho a ejecutar actos (en este caso, a emitir ruidos molestos) que de otro modo constituirían una nuisance. Con respecto a las dos primeras dificultades, señala que se trata más bien de dificultades prácticas que deberá remontar aquel que está alegando la prescripción. Por otra parte, agrega: dichas dificultades se ven atenuadas si se considera que para adquirir por prescripción en este

${ }^{21}$ En el Derecho inglés la adquisición por prescripción de un easement supone la existencia de un consentimiento tácito de parte del propietario del predio sirviente. Véase McFarlane (2008), p. 864 y ss. caso no es necesario demostrar que se han emitido ruidos molestos cada día durante el período de veinte años ${ }^{22}$. Es decir, la continuidad en el ejercicio del Derecho que se pretende adquirir por prescripción no se traduce en este caso en la necesidad de emitir ruidos molestos cada minuto de cada día durante el periodo de veinte años. Relativo a la tercera dificultad, señala que un inconveniente de similar naturaleza se observa en el caso de la adquisición por prescripción, por ejemplo, de un derecho de paso o de un derecho a descargar agua contaminada. En estos casos, la determinación de la precisa extensión de la servidumbre dependerá de diversos factores, lo cual hace posible que el titular goce de cierta latitud al respecto. Sin embargo, precisa, por regla general el titular del derecho a emitir ruidos molestos no debe gozar de similar latitud, en particular en razón del potencial efecto negativo que podría tener en el uso y goce del predio sirviente un aumento en los niveles de ruidos molestos. Pero, de nuevo, ello no se traduce en sostener la imposibilidad de adquirir por prescripción el derecho a emitir ruidos molestos.

Finalmente, en cuarto lugar, concluye que la verdadera razón por la cual corresponde sostener que los demandados no adquirieron por pres-

${ }^{22}$ Lo clave es que haya existido lo que podría calificarse como un ejercicio consistente de un derecho. McFarlane (2008), p. 866. Parece evidente que una noción de tal naturaleza se hace necesaria desde el momento en que se admite la posibilidad de adquirir por prescripción el derecho a ejecutar actos (como la emisión de ruidos molestos) que no es preciso ejecutar día a día. 
cripción el derecho a ejecutar actos que de otro modo constituirían nuisance (la emisión de ruidos molestos), no reside en el hecho de existir, dentro del requerido período mínimo de 20 años, un intervalo de dos años durante los cuales no se llevaron a cabo eventos generadores de ruidos molestos. Esa fue la razón esgrimida por el juez de primera instancia después de establecer que en el periodo que se extiende entre los años 1976 y 2009, durante los años 1991 y 1992 no hubo eventos de ningún tipo (ni en el estadio, ni en la pista o track). Sobre esto señala que lo esencial en un caso de prescripción de una servidumbre real como la que aquí se discute es establecer si la naturaleza y nivel de actividad por parte del titular del supuesto predio dominante a lo largo de un periodo mínimo de veinte años, tomada dicha actividad como un todo, fue suficiente como para hacer saber a cualquier persona razonable puesta en el lugar del dueño del supuesto predio sirviente que se estaba haciendo valer un derecho de carácter continuo (el derecho a emitir ruidos molestos), el cual, en caso de oposición a dicha pretensión, correspondía cuestionar. De esta forma, razona lord David Neuberger, el intervalo de dos años que tuvo lugar en este caso no constituía un problema insuperable para los demandados. No obstante dicho intervalo, era posible sostener que, en lo sustancial, los demandados, en forma consistente y continua, habían llevado a cabo actividades o eventos en el estadio y en la pista o track a lo largo de un periodo de al menos de veinte años. No obstante lo anterior, señala que la prescripción alegada por los demandados no puede prosperar por cuanto no se demostró que la actividad llevadas a cabo en el estadio y en la pista constituyeran una nuisance durante el período requerido de veinte años, puntualizando que no es suficiente demostrar que las actividades respectivas (en este caso, los eventos de carreras de velocidad) hayan sido ejecutadas o hayan creado ruido durante veinte años o más. Es necesario demostrar que tales actividades han sido constitutivas de nuisance, es decir, que han creado ruidos molestos durante un periodo mínimo de veinte años ${ }^{23}$. Y como ello no se probó suficientemente en este caso, la prescripción alegada no puede prosperar.

\section{b) En cuanto a la posibilidad} de alegar como defensa que el demandante "vino a la nuisance" El punto aquí consiste en determinar si el demandado puede esgrimir como defensa la circunstancia de que se encontraba ejecutando las actividades de las que se reclama desde antes de que el demandante arribara y se radicara en el lugar. Con arreglo al precedente en esta materia ${ }^{24}$, la respuesta es negativa, incluso si se demuestra que el demandante sabía de la actividad del demandado desde antes de arribar y radicarse en el lugar.

La decisión de la Corte en esta materia se sujetó a lo que señalaba el

${ }^{23}$ Ello presenta, por cierto, dificultades de prueba. Pero dichas dificultades no parecen irremontables si se recuerda que en este caso no era necesario probar que se habían emitido ruidos molestos todos los días durante el período de veinte años.

${ }^{24}$ Bliss v Hall (1838); Sturges v Bridgman (1879); Miller v Jackson (1977). 
precedente. Lord David Neuberger señaló que de acuerdo con el Case Law (Derecho de Casos), la no disponibilidad de tal defensa para el demandado es el principio que rige en esta materia. Dicha conclusión aparece reforzada en este caso, añade, por la circunstancia de que el uso dado por los demandados a su inmueble (un uso habitacional) es el mismo que el que había sido dado por sus predecesores, incluso desde antes que comenzara la emisión de ruidos molestos (debe recordarse aquí que "Fenland" había sido construida en la década de 1950).

Es interesante notar que la Corte no se limitó a rechazar la defensa sobre la base del precedente, sino que, además, incursionó brevemente en las razones que justifican este principio. Al respecto lord David Neuberger señaló que dicho principio se funda en la noción de que el tort de private nuisance es en términos concretos un tort en contra del inmueble, de tal manera que la posibilidad de reclamar en contra de los actos constitutivos de nuisance está determinada por el hecho de ser el demandante titular de un derecho propietario sobre el inmueble. Luego, continúa el razonamiento, no sería compatible con esa concepción el aceptar que un demandado que ha incurrido en actos constitutivos de nuisance sobre un inmueble, dejara de ser responsable por el sólo hecho de que el titular de derechos propietarios sobre dicho inmueble pasara a ser una persona distinta, aun cuando el uso que el nuevo titular diera al inmueble fuera el mismo que el de su predecesor ${ }^{25}$.

${ }^{25}$ Además, de admitirse la defensa de "venir a la nuisance", se estarían desconociendo las
Por otra parte, y a propósito del análisis del punto relativo a la noción de "venir a la nuisance", la Corte consideró una cuestión que parece presentar algunas similitudes, pero que en esencia es diferente. Supóngase que el demandante efectuó alteraciones en su inmueble (construcciones o cambios de uso) con posterioridad al inicio por parte del demandado de actividades en cuestión y que solo en virtud de dichas alteraciones las actividades del demandado pasaron a ser constitutivas de nuisance. Es decir, las actividades del demandado (actividades preexistentes: iniciadas antes de las alteraciones llevadas a cabo por el demandante) no habrían pasado a ser constitutivas de nuisance de no haber sido sino por las alteraciones llevadas a cabo por el demandante. El punto a dilucidar es si en tal caso puede prosperar una acción en private nuisance. Aunque lord David Neuberger señaló que no era necesario decidir ahora tal cuestión, sí puntualizó que las actividades preexistentes del demandado deberían considerarse como parte del carácter de la localidad o de la vecindad, en razón precisamente de que antes de las transformaciones llevadas a cabo por el demandante dichas actividades no eran constitutivas de nuisance. Por ende, por regla general, y en la medida que las actividades preexistentes del demandado reúnan ciertas condiciones, dichas actividades

normas relativas a los plazos de prescripción. En efecto, el demandado estaría en condiciones de derrotar al demandante por el solo hecho de haber arribado primero al lugar sin importar el número de años durante los cuales ha estado ejerciendo la actividad constitutiva de nuisance: Murphy (2010), p. 106. 
no darán lugar responsabilidad en private nuisance, no obstante que producto de la transformación llevada a cabo por el demandante pasen a ser constitutivas de nuisance.

c) En cuanto a la posibilidad de que el demandado invoque el uso que él hace de su inmueble o instalaciones para los efectos de determinar lo que se denomina "el carácter de la localidad"

Ya se ha dicho antes en este comentario que el núcleo esencial del tort de private nuisance está constituido por la existencia de una interferencia sustancial con el uso y goce de un inmueble. Solo aquellas interferencias que sean sustanciales serán accionables en private nuisance. Por ello cobra relevancia determinar cuándo una interferencia es sustancial. En este caso resulta aplicable el test de la razonable tolerabilidad por parte del demandante: en presencia de una interferencia, lo que se debe analizar es si cabe esperar razonablemente que ella sea tolerada por el demandante en toda circunstancia. Si la respuesta es negativa, la interferencia será sustancial.

Entre los factores ${ }^{26}$ a considerar en la aplicación de dicho test se encuentra el de la "naturaleza" o "carácter" de la localidad, factor que parece ser aplicable predominantemente en el caso de interferencias con lo que se ha denominado valor de amenidad

${ }^{26}$ Otros factores a considerar son, por ejemplo, la existencia de malicia por parte del demandado, el carácter especialmente sensitivo del demandante; la hora, duración, regularidad e intensidad de la interferencia, etc. McBride y Bagshaw (2012), pp. 422-434. del inmueble ${ }^{27}$. Así, es dable sostener que si la interferencia consiste, por ejemplo, en ruidos emitidos por una empresa metalmecánica, una persona residente en un barrio industrial, en el que abundan dichas empresas, tendrá más dificultades para establecer que tal interferencia es sustancial que las que tendría una persona residente en un barrio no industrial, en el que no existen otras empresas similares.

De esta forma, el punto a dilucidar aquí es si en la determinación del carácter de la localidad se deben tomar o no en consideración los usos o actividades de los demandados. En este caso en concreto, las alternativas parecían ser:

a) Definir el carácter de la localidad señalando que se trataba simplemente de una zona agrícola o

b) Definir el carácter de la localidad señalando que se trataba de una zona agrícola en la que existe un estadio y una pista que albergan periódicamente determinados eventos generadores de niveles de ruido como aquellos producidos por los demandados.

En esta parte lord David Neuberger, quien prefiere hablar de los "patrones establecidos de uso" en la localidad, comienza precisando que la evaluación de tal factor es una cuestión de hecho que, sin embargo, puede en-

${ }^{27}$ Se encuentra bien establecido que en el caso de daño físico al inmueble dicho daño es revelador por sí solo del carácter sustancial de la interferencia, por lo que no es necesario considerar el factor relativo a la naturaleza de la localidad: St Helen's Smelting Co v Tippings (1865). 
volver cuestiones de principio respecto de la cuales la Corte está en condiciones de ofrecer criterios a seguir.

Fija un criterio fundamental en esta materia. Señala que, si bien en principio se debe considerar la situación factual existente en el lugar para definir su carácter (en este caso, la presencia de un estadio y una pista o track), si las actividades del demandado son constitutivas de nuisance, ellas no deben ser consideradas para los efectos de definir el carácter de la localidad o "los patrones establecidos de uso" en la localidad. La razón es simple: solo actividades lícitas pueden ser consideradas en tal determinación. De esta forma, en este caso en concreto la alternativa correcta no es ninguna de las dos señaladas anteriormente, sino que una tercera: definir el carácter de la localidad señalando que se trata de una zona agrícola en la que existe un estadio y una pista destinados a albergar eventos generadores de ruido que no sean constitutivos de nuisance, es decir, que estén limitados a ciertos niveles considerados aceptables.

Por cierto, se podría sostener que si tales actividades se han venido desarrollando durante años, es intuititivo pensar que ellas han pasado a ser parte del carácter de la localidad. Sin embargo, lord David Neuberger señala que el criterio rector en esta materia no se ve alterado por el hecho de que las actividades de los demandados hayan tenido lugar durante varios años En tanto se trate de actividades constitutivas de nuisance, por ende, ilícitas, ellas no pueden ser consideradas en la determinación del carácter de la localidad, siendo irre- levante el que ellas se hayan venido practicando durante años.

Obviamente, añade: lo dicho reconoce una excepción en el caso que haya operado una prescripción adquisitiva del derecho a ejecutar actividades que de otro modo serían constituivas de nuisance. En tal caso las actividades, aún siendo constitutivas de una nuisance, deberán ser tomadas en consideración al definir el carácter de la localidad. Asimismo, dichas actividades (ilícitas, en tanto son constituivas de nuisance) deberán ser consideradas si, habiendo sido ellas objeto de una acción en private nuisance en un tiempo anterior, el tribunal respectivo, no obstante haber establecido que se incurrió en una nuisance, decidió no otorgar una injunction (es decir, no ordenó el cese de la actividad) sino que, en lugar de ella, una indemnización de perjuicios. Lord David Neuberger señala que en este caso se debe entender que dicha actividad ha sido en cierto modo "confirmada" o "ratificada" por el tribunal.

$\mathrm{El}$ criterio expuesto por lord $\mathrm{Da}$ vid Neuberger parece inobjetable. Sin embargo, está expuesto a la crítica de que parece incurrir en una cierta petición de principios. En efecto, si la determinación del carácter de la localidad es parte del proceso conducente a establecer si una actividad en particular es constitutiva de nuisance, no parece lógico excluir de antemano dichas actividades al efectuar la referida determinación, sobre la base de que ellas constituyen una nuisance, lo cual es precisamente lo que se está tratando de establecer.

Si bien reconoce que su razonamiento está sujeto a esta crítica (él 
habla de la existencia de un "elemento de circularidad" en el razonamiento), defiende la mantención del mismo sobre la base de tres puntos:

i) En la mayoría de los casos, aun antes de determinar el carácter de la localidad, será bastante claro si las actividades de las que se reclama constituyen o no efectivamente una nuisance. En tales casos, en consecuencia, no será necesario proceder a determinar el carácter de la localidad;

ii) En un pequeño número de casos existirá, sin embargo, según sus términos, un "elemento de circularidad". En tales casos la corte deberá emplear un proceso que él denomina "iterativo": sobre la base de ciertos usos establecidos en la localidad corresponderá preguntarse sucesivamente si determinados niveles de ruidos son o no aceptables, hasta llegar a un nivel sobre el cual los ruidos se considerarán molestos o inaceptables y

iii) En todo caso, las opciones alternativas al criterio sugerido (conforme al cual se hace una distinción en torno a la naturaleza de las actividades del demandado) no están exentas de dificultades.

$\mathrm{Si}$ se optara por ignorar totalmente las actividades de los demandados para los efectos de determinar el carácter de la localidad, incluso si se trata de actividades lícitas, ello significaría en algunos casos una notoria injusticia para el demandado. Y si, a la inversa, se optara por tomar siempre en consideración las actividades del demandado para determinar el carácter de la localidad, incluso si se trata de actividades constitutivas de nuisance, sería casi imposible que alguna vez prosperara una acción en nuisance: si dichas actividades fueran parte del carácter de la localidad, no se divisa cómo se podría concluir que ellas son inaceptables después de aplicar como estándar precisamente ese carácter. Ello no solo revela una petición de principios (o "circularidad") de alcances mucho más inaceptables sino que, también, implica permitir que el demandado en definitiva se beneficie de su propia actividad ilícita, algo que el Derecho siempre ha repudiado.

d) En cuanto a la incidencia de la existencia de una autorización administrativa (planning permission) otorgada al demandado con respecto a un uso del suelo específico en la determinación de si existió o no una nuisance

Esta materia fue la más relevante en el pleito. Debe recordarse que los demandados contaban con un conjunto de autorizaciones administrativas que los habilitaba para albergar en el estadio y en la pista los eventos generadores de ruidos molestos, lo que a primera vista aparece como un obstáculo insalvable para establecer su responsabilidad civil. Y es a propósito de esta materia en donde la decisión de la Corte Suprema implicó un significativo desarrollo en materia de las normas aplicables a private nuisance.

En teoría, el otorgamiento de una autorización administrativa (planning per- 
mission) sobre un determinado uso del suelo puede incidir en dos aspectos:

i) El demandado podría sostener que procede rechazar la demanda por cuanto el uso por el que se reclama es precisamente el uso autorizado (en este caso, la emisión de ruidos molestos). Existiría lo que podría llamarse una autorización directa de una private nuisancey

ii) El demandado podría sostener que en virtud de la autorización administrativa ha operado un cambio en la naturaleza o carácter de la localidad, en la medida que ella permite que el inmueble del demandado $\mathrm{u}$ otros inmuebles en la localidad puedan ser destinados a un determinado uso (en este la emisión de ruidos molestos).

En relación con lo expuesto en el primer punto, la Corte reafirmó el criterio establecido en anteriores decisiones judiciales ${ }^{28}$, en el sentido que una autorización administrativa no puede autorizar directamente la comisión de una private nuisance. El punto aquí es que el tort de private nuisance protege en contra de la violación de derechos propietarios, y si se entendiera que la decisión de la autoridad administrativa en materia de usos del suelo se traduce en una autorización para cometer una nuisance, ello significaría que dicha autoridad administrativa estaría privando de sus derechos propietarios al demandante sin otorgarle compensación alguna,

${ }^{28}$ Wheeler v JJ Saunders Ltd (1994); Hunter v Canary Wharf (1997). atribución que no le ha sido conferida a la autoridad administrativa por parte del Parlamento en la legislación relativa a usos del suelo.

Con respecto al segundo punto, hasta la dictación de este fallo el criterio parecía ser el establecido en Gillingham BC v Medway (Chatham) Dock Co Ltd (1991). Con arreglo a lo resuelto en Gillingham, se entendía que una autorización administrativa relativa al uso del suelo cambiaba la naturaleza o carácter de la localidad en la medida que dicha autorización tuviera relación con un área geográfica de cierta extensión, en términos tales que la autorización pudiera concebirse como "estratégica" o relativa a un desarrollo importante o de cierta envergadura. En tales casos, en consecuencia, al cambiar el carácter de la localidad, los usos o actividades autorizados no serían constitutivos de nuisance por ser concordantes con el nuevo carácter o naturaleza de la localidad ${ }^{29}$. A la inversa, se entendía que autorizaciones administrativas relativas solo a una pequeña área geográfica (por ejemplo, relativas solo al inmueble del demandado), no eran suficientes para estimar que había cambiado la naturaleza o el carácter de la localidad.

Es sobre este segundo punto que la Corte desarrolla las normas de private nuisance, por cuanto se apartó

${ }^{29}$ Aunque se admita que las autorizaciones administrativas no pueden autorizar una nuisance, están de igual forma sujetas a crítica puesto que en virtud de ellas la administración interfiere indirectamente con los derechos privados sin compensación, por la vía de alterar el carácter o naturaleza de la localidad: MCBRIDE y BaGshaW (2012), pp. 430-431. 
del precedente establecido en $\mathrm{Gi}^{-}$ llingham, señalando que no cabía hacer la distinción que allí se formulaba y que, por regla general, la existencia de una autorización administrativa es, por sí sola, irrelevante para determinar el carácter o naturaleza de la localidad y, por ende, para determinar si existió o no nuisance ${ }^{30}$. El carácter de la localidad, se dijo, cambia por lo que de hecho allí se ejecuta, más allá de que se cuente o no con una autorización administrativa relativa al uso del suelo, rigiendo aquí en consecuencia el criterio que se indicó cuando se analizó la posibilidad de considerar las actividades del demandado para evaluar un cambio en el carácter de la localidad (relativo a la licitud de tales actividades, sin perjuicio de los casos excepcionales que allí se indicaron).
Solo en el restringido marco fijado por dicho criterio es posible sostener que una autorización administrativa podría contribuir a determinar un cambio en la naturaleza o carácter de la localidad.

En cuanto a las razones para apartarse del precedente establecido en Gillingham, lord David Neuberger puntualizó la paradoja a la que conducía la distinción formulada en dicha decisión, por cuanto tal perspectiva implicaba sostener que mientras mayor fuera el probable impacto negativo del cambio de uso (por abarcar una

${ }^{30}$ Lord Robert Carnwath, sin embargo, sostuvo que en casos excepcionales (como una planificación "estratégica") una autorización administrativa por sí sola puede conducir a un cambio en el carácter de la localidad, por lo que no puede ser ignorada por el tribunal. Es decir, sostuvo que era correcto el razonamiento en Gillingham. Véase el párrafo 223 del fallo. mayor zona geográfica) más dificil sería para un demandante establecer que se había cometido una private nuisance. Además, sostiene que es incorrecto caracterizar las autorizaciones conferidas en el marco de lo que se llama "planificaciones estratégicas" (o de "mayor envergadura") como indicativas de que son ellas las que han considerado el interés publico, caracterización que serviría de fundamento para la distinción allí formulada. Más bien, argumenta, cabe suponer que toda autorización en materia de uso de suelo ("estratégica" o no) considera el interés público.

e) En cuanto a la incidencia

que la existencia de una autorización administrativa otorgada al demandado (para un específico uso de suelo) puede tener en la determinación del remedio a otorgar al demandante

En el punto anterior se analizó lo que dijo la Corte con respecto a la relevancia de la existencia de una autorización administrativa en la etapa en la que está determinando si existió o no una nuisance. En esta punto se analiza la incidencia de dicha autorización en una etapa posterior, una vez que ya se ha establecido que se ha cometido una nuisance. Se trata de la etapa en la que el tribunal, habiendo establecido que se cometió una nuisance, procede a determinar el remedio a otorgar al demandante. A propósito de este punto la Corte también desarrolla las normas de private nuisance en una manera significativa.

$\mathrm{Al}$ comienzo de este comentario se indicó que en materia de private nui- 
sance, dentro del conjunto de remedios disponibles (injunctions e indemnización de perjuicios), las injunctions ocupaban un papel relevante. En efecto, normalmente el demandante, además de pretender que se le indemnice por el daño ya causado, pretenderá en particular que se prevenga la comisión de futuras nuisances. Para ello buscará que se dicte una orden judicial (injunction) que prohiba al demandado la ejecución de la actividad constitutiva de nuisance $^{31}$.

Si se establece que se cometió una nuisance, el tribunal otorgará una indemnización de los perjuicios ya causados. Sin embargo, con respecto a la prevención de futuras nuisances, el tribunal, no obstante haber establecido que se cometió una nuisance, no necesariamente otorgará una injunction, sino que otorgará en su lugar (in lieu) una indemnización de perjuicios dirigida a compensar al demandante por interferencias sustanciales futuras. Como se puede ver, lo que en definitiva tiene lugar en tal caso es que el demandado pasará a estar autorizado para seguir ejecutando la actividad constitutiva de nuisance, en la medida que pague la indemnización fijada para compensar por las nuisances futuras. Como se dijo antes en este comentario, en cierta forma el tribunal en tal caso "ratifica" o "confirma" la actividad ejecutada por

${ }^{31}$ Se trata en tal caso de una injunction prohibitoria, que es la que es comúnmente pretendida por los demandantes en private nuisance. Otras clases de injunction son la mandatory injunctions, la quia timet injunction, y la interim injunction (lo que en el Derecho chileno sería una especie de medida precautoria): MCBRIDE y BAGSHAW (2012), pp. 454-455. el demandado, en el sentido de que no se prohibe que dicha actividad se siga ejecutando (sujeto, eso sí, al pago de la indemnización fijada).

Lo anterior hace necesario contar con un criterio con arreglo al cual decidir si corresponde otorgar una injunction o, bien, una indemnización en lugar de una injunction (para compensar interferencias sustanciales futuras). Hasta antes del fallo que ahora se comenta, dicho criterio se econtraba establecido en Shelfer v City of London Electric Lighting Co (1894). En Shelfer se señaló que se podía otorgar una indemnización compensatoria de interferencias sustanciales futuras en lugar de una injunction, en la medida que concurrieran los siguientes requisitos copulativos:

i) si la lesión de los derechos legales del demandante es pequeña;

ii) si dicha lesión es suceptible de ser estimada en dinero;

iii) si dicha lesión es suceptible de ser compensada adecuadamente mediante el pago de una pequeña suma de dinero; $y$

iv) si se trata de un caso en el que sería opresivo para el demandado el otorgamiento de una injunction.

Sobre la regla establecida en Shelfer, tres puntos cabe destacar. En primer lugar, en posteriores decisiones judiciales se clarificó que también era posible otorgar una indemnización por interferencias futuras en lugar de una injunction en otros casos que no quedaran comprendidos en la regla (por ejemplo, en caso que el demandante dejara transcurrir un tiempo excesivo antes de buscar el otorgamiento de 
una injunction) $)^{32}$. En segundo lugar, en la regla de Shelfer no aparece la noción de interés público como una factor para fundamentar la decisión de rechazar una injuction y otorgar en su lugar una indemnización por interferencias futuras (en el sentido de que en razón del interés publico envuelto, procede "autorizar" que el demandado continue desarrollando la actividad constitutiva de nuisance). Aunque en ocasiones se discutió, la irrelevancia de la noción de interés publico en esta materia parecía ser la regla establecida ${ }^{33}$. Y, en tercer lugar, considerando los requisitos establecidos en Shelfer (a pesar del carácter abierto de la regla) y la recién señalada irrelevancia en general de la noción de interés público, queda en evidencia la cautela con la que procederían los tribunales para los efectos de rechazar una injunctiony otorgar en su lugar una indemnización por interferencias futuras, al extremo de que se llegó a decir que ello tendría lugar en muy excepcionales circunstancias y cuando fuera verdaderamente opresivo otorgar la injunction $^{34}$. En otras palabras, bajo la regla Shelfer se podía decir que la regla general era el el otorgamiento de injunctions. Dicha cautela parecía estar justificada por lo que implicaba el rechazo de la injunction: una verdadera "autorización" para que el demandado siguiera cometiendo la nuisance.

En esta materia la Corte se apartó en cierta forma de lo que parecía

${ }^{32}$ McBride y Bagshaw (2012), p. 456.

${ }^{33}$ En Miller v Jackson (1977) se rechazó la injunction en virtud de razones de interés publico. En KenNaWAY v THOMPSON (1980) la decisión se sujetó a la regla establecida en Shelfer.

${ }^{34}$ Watson v Croft Promosport Ltd (2009). ser la regla establecida. Si bien lord David Neuberger señaló que una vez establecida una nuisance, el remedio a otorgar es prima facie una injunction (es decir, se mantiene como regla general el otorgamiento de una injunction), también dejó establecido que había dos problemas en relación con lo que dicen los precedentes en esta materia:

i) Respecto de la verdadera naturaleza del test establecido en Shelfer (si tiene un carácter exclusivo o si también cabe considerar otros factores, tales como la conducta de las partes) y

ii) En relación con la forma en que la noción de interés público debe ser tomada en cuenta (en el sentido que cabe considerarlo solo cuando la lesión a los derechos del demandante es menor).

En cuanto al primer problema, lord David Neuberger señaló que la regla en Shelfer "no debía atar de manos" a los jueces, ni ser una regla que se aplicara mecánicamente; sugiriendo, además, que la aproximación a esta debe ser más flexible. Por ello no duda en calificar como errónea la aseveración de que solo en muy excepcionales circunstancias procederá el rechazo de una injunction. En otras palabras, de acuerdo con lo señalado por lord David Neuberger, los jueces deben gozar de mayor discreción para determinar si corresponde rechazar el otorgamiento de una injunction. Indica, asimismo, que prima facie, establecida una nuisance, corresponde otorgar una injunction, pero ello no obsta a que los jueces dispongan de 
mayor libertad para considerar otros factores (no solo los mencionados en la regla Shelfer) y decidir si corresponde rechazar o no el otorgamiento de tal medida, sin mostrarse inclinados de antemano por una u otra alternativa. En ausencia de esos factores adicionales, será suficiente que se cumplan los requisitos de la regla Shelfer para rechazar una injunction. Pero ello no implica aseverar que si no se satisfacen todos esos requisitos deberá necesariamente otorgarse una injunction. En este contexto, señala, es de cargo del demandado demostrar el por qué procede rechazar la injunction solicitada ${ }^{35}$.

En cuanto al segundo problema, lord David Neuberger estableció que la noción de interés público es siempre relevante, por lo que dicha noción deberá ser un factor a ser tomado en cuenta para los efectos de decidir acerca del eventual rechazo de una injunction. En este contexto, agrega, la existencia de una autorización administrativa en materia de usos de suelo podría justificar el rechazo de una injunction, en razón del interés público que envuelve siempre una autorización de tal naturaleza. En todo caso, la existencia de dicha autorización es uno más de los factores a considerar.

De esta forma, si bien se mantiene el principio de que establecida una

${ }^{35}$ Lord Jonathan Sumption sostuvo una posición más categórica, por cuanto señaló que la regla en Shelfer estaba "caduca" y que lo que correspondía era afirmar que la regla general en materia de remedios no debía ser el otorgamiento de una injunction sino que el otorgamiento de una indemnización de perjuicios. Véase al respecto el párrafo 161 del fallo. nuisance procede, como regla general, el otorgamiento de una injunction, la Corte flexibiliza en forma significativa la aplicación de la regla en Shelfer e introduce la noción de interés publico como un factor que siempre se debe tener en consideración, por lo que en el caso particular de una autorización administrativa para el uso de suelo, su existencia puede justificar el rechazo del otorgamiento de una injunction (en razón del interés público que supone el otorgamiento de dicha autorización) $)^{36}$.

\section{Conclusiones}

El análisis de la sentencia comentada sugiere al menos tres ideas.

- En primer lugar, el desarrollo de las normas de private nuisance por parte de la Corte incide en problemas particularmente relavantes en la vida moderna. La disputa respecto de los usos que se debe dar a suelos cada vez más escasos, producto del crecimiento de la población y de la necesidad de expansión de la infraestructura industrial o de servicios de un país; el papel de la autoridad administrativa en la resolución de esas disputas mediante la determinación de los usos del suelo y el otorgamiento de autorizaciones para usos específicos; los conflictos que tales disputas generan,

${ }^{36}$ El fallo, además, dejó abierto el tema relativo a la forma de determinar el monto de la indemnización por interferencias futuras. 
enfrentando los derechos de los individuos entre sí y los derechos de los individuos con el interés público, etc., son todas cuestiones en las que a primera vista pareciera que el derecho privado no tiene o no debería tener incidencia alguna. Intuitivamente alguien podría sentirse inclinado a pensar que se trata de materias que son del solo dominio de normas regulatorias de Derecho Administrativo y Ambiental. Sin embargo, el fallo objeto de este comentario demuestra lo contrario. Es dable sostener que en el derecho inglés las normas de Derecho Privado sobre private nuisance complementan las normas regulatorias de carácter administrativo para abordar problemas como los atrás descritos. De esta forma, la existencia de normas regulatorias de carácter administrativo no excluye de forma necesaria el papel en este campo de las normas de Derecho Privado. Por cierto, uno podría preguntarse si el derecho privado debería ocuparse o no de estas materias en conjunto con el derecho regulatorio. Pero antes de abordar esa cuestión normativa es preciso adoptar una perspectiva análitica: es necesario identificar el papel preciso que respecto de esas materias el derecho privado está en condiciones desempeñar en un sistema legal es- pecífico. Solo así se podrá evaluar la conveniencia o no de la incidencia del Derecho Privado en estas cuestiones.

- En segundo lugar, el análisis que ofrece la sentencia objeto de este comentario no debe considerarse como enteramente ajeno a nuestra realidad jurídica. En efecto, en el Derecho Civil francés, a propósito del cual el Derecho Civil chileno reconoce una especial cercanía, problemas como los abordados por las normas de private nuisance del Derecho inglés son enfrentados por una doctrina de creación judicial. Enfrentados a tales problemas, los tribunales franceses contaban solo con las normas sobre interdictos posesorios $\mathrm{y}$ sobre responsabilidad extracontractual contenidas en el Código de Napoleón. La insuficiencia de dichas normas determinó que a partir del siglo XIX la jurisprudencia francesa comenzara a desarrollar la doctrina denominada troubles de voisinage, la que ofrece paralelos significativos con las normas de private nuisance del Derecho inglés ${ }^{37}$.

- Y, en tercer lugar, lo recién dicho hace posible que uno pueda preguntarse si existe espacio en el Derecho chileno para desarrollar jurisprudencialmente algunas de las ideas que uno encuentra tanto en

\footnotetext{
${ }^{37}$ Véase al respecto Gordley (2010), pp.
} 1-28. 
las normas de private nuisance como en la doctrina francesa de los troubles de voisinage. A fin de responder esa pregunta es preciso exponer de forma previa las alternativas que el Derecho Privado chileno y su Código Civil ofrecen para abordar problemas como los resueltos en la sentencia comentada $^{38}$. Ello posibilitará no solo responder dicha pregunta sino que, también, proporcionará el material necesario para abordar la cuestión normativa planteada más atrás, la que también es posible formular sobre el del Derecho Privado chileno: si el Derecho Privado chileno debe tener alguna incidencia en estas materias, y en la afirmativa, en qué medida. El abordar estas cuestiones permitirá que, por ejemplo, aquella persona que quiera reclamar de ruidos molestos que interfieren con el uso y goce de su inmueble, esté en condiciones de saber si su pretensión, que se puede resumir en la exigencia de que el demandado "se calle", contará o no con el respaldo del a veces menospreciado, pero siempre útil Derecho Privado.

38 Destaca en este sentido Amunategui (2013), pp. 46-86, quien pretende sentar las bases para la formulación en el Derecho chileno de un concepto de lo que se denomina "inmisiones"

\section{BibLIOGRAFÍA CITADA}

Amunátegui, Carlos (2013). "Hacia un concepto de inmisiones en el derecho chileno". Revista Chilena de Derecho. V. $40 \mathrm{~N}^{\circ} 1$. Santiago.

Beever, Allan (2013). The Law of Private Nuisance. Oxford: Hart Publishing.

BELL, John (2013). 'Sources of Law', in Andrew Burrows (ed.). English Private Law. $3^{\text {a }}$ ed. Oxford: Oxford University Press.

Birks, Peter (1995). "The concept of a civil wrong", en David OwEN (ed). Philosophical Foundations of Tort Law. Oxford: Oxford University Press. Rep. 2001.

Gordley, James (2010). "Disturbances among neighbours: an introduction", in James GordLey. The development of liability between neighbours. Cambridge: Cambridge University Press.

Le Sueur, Andrew (2009). 'Constitutional Fundamentals: A. Fundamental Principles', in David Feldman (ed.). English Public Law. 2a ed. Oxford: Oxford University Press.

McBride, Nicholas y Roderick BAGSHAW (2012). Tort Law. 4th ed. Harlow: Pearson.

McFarlane, Ben (2008). The Structure of Property Law. Oxford: Hart Publishing.

MurPhy, John (2010). The Law of Nuisance. Oxford: Oxford University Press.

Nolan, Donal (2012). “'A tort against land': private nuisance as a property tort", in Donal Nolan y Andrew RoBERTSON (eds.). Rights and Private Law. Oxford: Hart Publishing. Rep. 2014.

Stevens, Robert (2007). Torts and Rights. Oxford: Oxford University Press. Rep 2009. 
Zweigert, Konrad y Kötz, Hein (1998). Introduction to Comparative Law. $3^{\text {rd }}$ ed. (trad.) Tony Weir. Oxford: Clarendon Press.

\section{Normas citadas}

Código Civil chileno.

Código Civil francés.

Constitutional Reform Act, de 2005.

\section{Jurisprudencia citada}

Bliss v Hall (1838): Court of Common Pleas, 17 de enero de 1838 (demanda por el tort de private nuisance), (1838) 4 Bing (NC) 183.

Gillingham BC v Medway (Chatham Docks) Co Ltd (1991): Queen's Bench Division, 31 de julio de 1991 (demanda por el tort de private nuisance), [1993] QB 343.

Hunter and others v Canary Wharf Ltd (1997): House of Lords, 24 de abril de 1997 (demanda por el tort de private nuisance), [1997] AC 655.

Kennaway v Thompson (1980): Court of Appeal (Civil Division), 30 de abril

de 1980 (demanda por el tort de private nuisance), [1981] QB 88 (CA).

Miller v Jackson (1977): Court of Appeal (Civil Division), 6 de abril de 1977 (demanda por el tort de private nuisance), [1977] QB 966 (CA).

St Helen's Smelting Co v Tipping (1865): House of Lords, 5 de julio de 1865 (demanda por el tort de private nuisance), (1865) 11 ER 1483 (HL).

Shelfer v City of London Electric Lighting Company (1894): Court of Appeal, 18 de diciembre de 1894 (demanda por el tort de private nuisance), [1895] 1 Ch 287.

Sturges v Bridgman (1879): Court of Appeal, 1 de julio de 1879 (demanda por el tort de private nuisance), (1879) 11 Ch D 852 (CA).

Watson v Croft Promo-Sport Ltd (2009): Court of Appeal (Civil Division), 26 de enero de 2009 (demanda por el tort de private nuisance), [2009] EWCA Civ 15.

Wheeler v JJ Saunders Ltd (1994): Court of Appeal (Civil Division), $19 \mathrm{de}$ diciembre de 1994 (demanda por el tort de private nuisance), [1996] Ch 19 (CA). 